\title{
Induced Deficits in Speed Perception by Transcranial Magnetic Stimulation of Human Cortical Areas $\mathrm{V} 5 / \mathrm{MT}+$ and $\mathrm{V} 3 \mathrm{~A}$
}

\author{
Declan J. McKeefry, ${ }^{1}$ Mark P. Burton, ${ }^{1}$ Chara Vakrou, ${ }^{1}$ Brendan T. Barrett, ${ }^{1}$ and Anthony B. Morland ${ }^{2}$ \\ ${ }^{1}$ School of Optometry and Vision Science, University of Bradford, Bradford, West Yorkshire BD7 1DP, United Kingdom, and ${ }^{2}$ York Neuroimaging Centre, \\ Department of Psychology, University of York, York Y010 5DD, United Kingdom
}

\begin{abstract}
In this report, we evaluate the role of visual areas responsive to motion in the human brain in the perception of stimulus speed. We first identified and localized V1, V3A, and V5/MT + in individual participants on the basis of blood oxygenation level-dependent responses obtained in retinotopic mapping experiments and responses to moving gratings. Repetitive transcranial magnetic stimulation (rTMS) was then used to disrupt the normal functioning of the previously localized visual areas in each participant. During the rTMS application, participants were required to perform delayed discrimination of the speed of drifting or spatial frequency of static gratings. The application of rTMS to areas V5/MT and V3A induced a subjective slowing of visual stimuli and (often) caused increases in speed discrimination thresholds. Deficits in spatial frequency discrimination were not observed for applications of rTMS to V3A or V5/MT + . The induced deficits in speed perception were also specific to the cortical site of TMS delivery. The application of TMS to regions of the cortex adjacent to V5/MT and V3A, as well as to area V1, produced no deficits in speed perception. These results suggest that, in addition to area V5/MT+, V3A plays an important role in a cortical network that underpins the perception of stimulus speed in the human brain.
\end{abstract}

Key words: transcranial magnetic stimulation; fMRI; retinotopic mapping; psychophysics; speed perception; V5/MT+; V3A

\section{Introduction}

Neuroimaging studies have shown that there is an extensive network of cortical areas that are responsive to moving stimuli in the human brain (Watson et al., 1993; Dupont et al., 1994; Orban et al., 1995; Tootell et al., 1995a,b; McKeefry et al., 1997; Huk and Heeger, 2000). Without doubt, the most intensively studied of these is area $\mathrm{V} 5 / \mathrm{MT}+$, whereas, by comparison, other areas of the brain that respond to motion have received much less interest. One such area, V3A, has been found to be second only to V5/ $\mathrm{MT}+$ in terms of its sensitivity to motion (Tootell et al., 1997; Smith et al., 1998; Seiffert et al., 2003), but little is known about the exact nature of its contribution to motion perception. Similarly, the study of the neural mechanisms that underpin the perception of speed has also tended to focus on the role played by V5/MT. In nonhuman primates, V5/MT neuronal activity encodes speed information (Maunsell and Van Essen, 1983; Rodman and Albright, 1987; Lagae et al., 1993; Perrone and Thiele, 2001; Priebe et al., 2003; Priebe and Lisberger, 2004; Liu and Newsome, 2005, 2006; Nover et al., 2005; Krekelberg et al., 2006a,b) and lesions to this area bring about deficits in speed

Received May 1, 2008; revised May 1, 2008; accepted May 21, 2008.

This work was supported by Biotechnology and Biological Sciences Research Council Grant BB/E00413X/1. We thank Dr. Alexander Sack and his colleagues in the Faculty of Psychology at Maastricht University for their help and advice with the fMRI-guided TMS system. We also thank Uinsionn 0'Breathnach, Andre Gouws, and the staff of the York Neuroimaging Centre for their assistance in the scanning sections of these experiments.

Correspondence should be addressed to Declan J. McKeefry, School of Optometry and Vision Science, School of Life Sciences, University of Bradford, Bradford, West Yorkshire BD7 1DP, UK. E-mail: d.mckeefry@bradford.ac.uk. DOI:10.1523/JNEUROSCI.1287-08.2008

Copyright $\odot 2008$ Society for Neuroscience $\quad$ 0270-6474/08/286848-10\$15.00/0 perception (Newsome and Paré, 1988; Orban et al., 1995). In the human brain, speed-dependent activity has been reported in V5/ MT+ (Chawla et al., 1998; Huk and Heeger, 2000), but not consistently across studies (Sunaert et al., 2000). But the roles played by other motion-sensitive areas in the perception of speed, in particular V3A, remain mostly unexplored.

This study examines the functional organization of speed processing in the human brain, in particular the contributions made by visual areas V5/MT+ and V3A. To this end, we deployed repetitive transcranial magnetic stimulation (rTMS) to transiently disrupt the neural activity in these areas while observers perform a delayed speed discrimination task. We identified our targets for TMS functionally, in terms of their activation by motion. Importantly, we also used retinotopic mapping to disambiguate the location of $\mathrm{V} 3 \mathrm{~A}$ and $\mathrm{V} 1$ from neighboring retinotopic representations in the occipital lobe.

TMS has been widely applied to the study of various aspects of human brain function (Pascual-Leone et al., 2000; Walsh and Cowey, 2000). Motion perception in particular has proven to be very amenable to study using TMS (Beckers and Hömberg, 1992; Hotson et al., 1994; Beckers and Zeki, 1995; Anand et al., 1998; Walsh et al., 1998; Cowey et al., 2006; Laycock et al., 2007). However, thus far, only one study has specifically examined the effects of TMS to human area V5/MT+ on the perception of speed (Matthews et al., 2001) and demonstrated that the application of TMS to the lateral occipital cortex can impair performance on speed discrimination tasks. In this study, we use detailed functional magnetic resonance imaging (fMRI) mapping of individ- 
ual brains to guide the delivery of TMS not only to V5/MT + , but to other cortical areas such as V3A, to assess their contribution to the perception of stimulus speed.

\section{Materials and Methods}

MRI imaging and analysis. T2* magnetic resonance $(\mathrm{MR})$ images were acquired during visual stimulation using a GE Head Excite 3T MRI system at the York Neuroimaging Centre. A multislice two-dimensional gradient echo-planar imaging sequence [repetition time (TR), 3000 ms; echo time (TE), $25 \mathrm{~ms} ; 128 \times 128 \times 39$ matrix; in-plane field of view (FOV), $288 \times 288$ $\mathrm{mm}$ field of view; interleaved slice order with no gap] was used to measure the blood oxygenation level-dependent (BOLD) signal as a function of time. Every $3 \mathrm{~s}, 39$ 3-mm-thick slices were acquired axially for a duration of $252 \mathrm{~s}$ (seven stimulus cycles of $36 \mathrm{~s}$ each), yielding 84 temporal samples. A high-resolution T1-weighted three-dimensional anatomical data set was used for coregistration.

Functional and retinotopic identification of $v i$ sual areas responsive to motion. We performed detailed functional and retinotopic mapping of the occipital cortex using fMRI techniques in each of the five participants in the study. In terms of functional classification, regions of the brain responsive to moving visual stimuli were identified using a block-design paradigm in which brain activations generated by a moving stimulus were contrasted with those elicited by a static version of the same stimulus. The stimulus itself comprised a sinusoidal luminance grating of spatial frequency equal to $1 \mathrm{c} /{ }^{\circ}$ with a contrast of $50 \%$, subtending $30^{\circ} \times 25^{\circ}$. In the moving phase of the experiment, the grating moved at a speed of $8 \% \mathrm{~s}$ in a rightward direction for $3 \mathrm{~s}$, followed by a leftward direction for the same period. In the stationary phase, the grating remained static for $6 \mathrm{~s}$. Throughout the whole of the scanning procedure, observers were instructed to fixate on a centrally placed cross.

The fMRI data obtained with the moving versus stationary grating paradigm were analyzed with the BrainVoyager QX software (version 2.0) (Brain Innovation). Preprocessing of the data included threedimensional motion correction, spatial smoothing with a $4 \mathrm{~mm}$ Gaussian kernel (full width at half-maximum), linear trend removal, and highpass filtering at $0.01 \mathrm{~Hz}$. The statistical analysis of the BOLD signal was performed with multiple linear regression. The time course of the BOLD signal was regressed on a box-car function that followed the motion and stationary conditions of the experiment. This function was modified to account for the nature of the hemodynamic response (Boynton et al., 1996).

Figure 1 shows the regions of the brain that were most responsive to the motion versus stationary comparison in two representative observers (M.P.B., N.C.). In all cases, the comparison of the motion versus stationary condition generated significant bilateral activations in regions consistent with previously reported locations for area V5/MT+ (Tootell et al., 1995a), on the lateral surface of the occipito-temporal cortex (Fig. 1, top panels). Bilateral activations in the superior occipito-parietal regions of the cortex were also elicited by visual motion (Fig. 1, bottom panels). These activations are in the vicinity of area V3A (Tootell et al., 1997), but to demonstrate definitively that these activations did indeed fall within the full hemifield representation that occurs within V3A, we compared the functional results with those from retinotopic mapping procedures.

The multiple representations of the central visual field that exist in the occipital cortex were mapped in the same five observers using a standard previously identified as V3A.
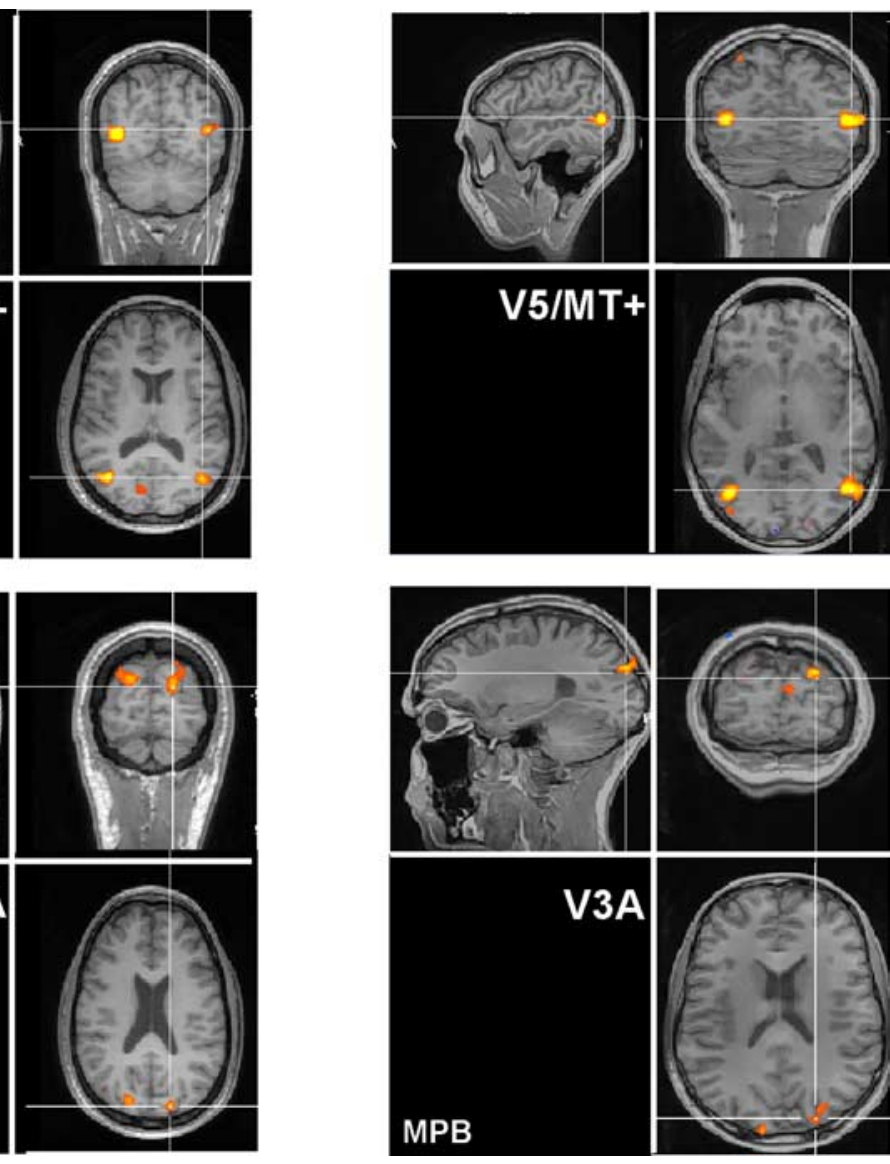

MPB

Figure 1. Functional identification of cortical areas V5/MT + and V3A. Regions of the brain that are most significantly activated by the passive viewing of moving gratings (see Materials and Methods for details) are shown. Areas of most significant ( $p_{\text {(uncorrected })}<0.0005$ ) are shown for two representative observers (N.C., M.P.B.). There is bilateral activation of the

paradigm incorporating rotating wedge and expanding ring stimuli in separate fMRI sessions. These stimuli create traveling waves of neural activation in the cortex, and for each voxel the phase of the maximum activation provides an indication as to the region of the visual field to which that part of the cortex is responsive (Sereno et al., 1995; Engel et al., 1997). The visual stimuli were formed from parts of the circular checkerboard pattern, which comprised eight concentric circles and 24 sectors. The full pattern had a radius of $14^{\circ}$, so individual checks were $1.66^{\circ}$ in radius. Two stimulus regimens were used: expanding rings and rotating wedges. The ring stimuli were formed by presenting three neighboring circles at any one time, starting with the three most central rings. The rings moved cyclically through the eight possible positions in a period of $36 \mathrm{~s}$, so the original central stimulation resumed after one stimulus cycle. The wedge was one-quarter of the full circle and rotated anticlockwise to resume its original position after $36 \mathrm{~s}$. Both the ring and wedge stimuli underwent pattern reversal at $6 \mathrm{~Hz}$; that is, each check was shown for 83 $\mathrm{ms}$, before reversing contrast polarity. The checks were high contrast of $>98 \%$ and were presented on a uniform gray of a luminance equivalent to the mean of the checkerboard. For our procedures, we presented eight stimulus cycles of rings and wedges during separate fMRI imaging acquisitions.

T1-weighted MR images (TR, $6.88 \mathrm{~ms}$; TE, $2.94 \mathrm{~ms} ; 176 \times 256 \times 256$ matrix; FOV, $289 \times 289 \mathrm{~mm}$; in-plane resolution, $1.13 \times 1.13$; slice thickness, $1 \mathrm{~mm}$ ) were used to create a flattened representation of the cortical gray matter (Teo et al., 1997; Wandell et al., 2000). After registration of the $2^{\star}$-weighted images to the coordinate frame of the T1weighted images, the fMRI time series (TS) was projected onto the flat- 
a)
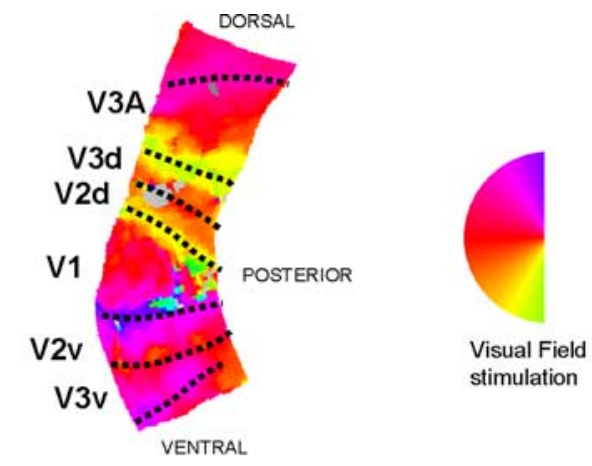

b)

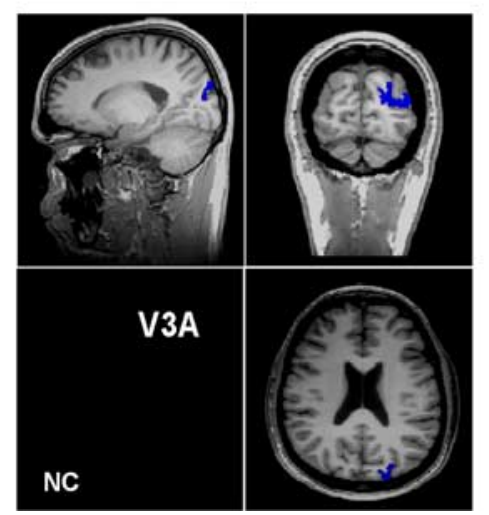

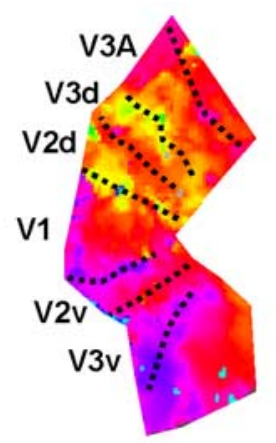

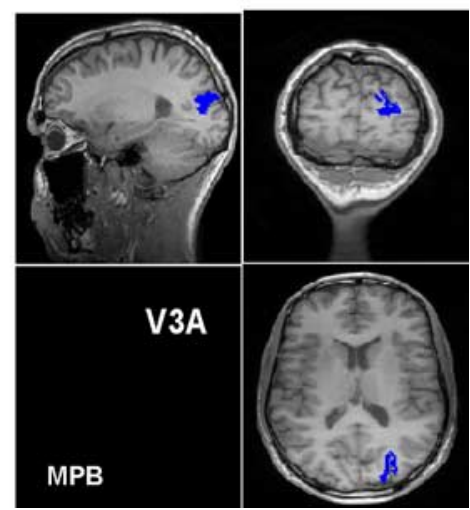

Figure 2. Retinotopic validation of functionally defined area V3A. $\boldsymbol{a}$, The top panel shows the BOLD response phase depicted in false color on the flattened representations of the right hemisphere occipital gray matter for two observers (N.C., M.P.B.). The broken lines indicate locations of visual area boundaries, which occur at reversals in the phase distributions. The inserted representation of the left hemifield allows a direct comparison between the BOLD phase and the region of the visual field stimulated. $\boldsymbol{b}$, The bottom panel shows the areas identified as V3A on the flattened maps represented on the three-dimensional volume structural MRIs (shown in blue). If one compares these retinotopic localizations of area V3A, with those motion activations shown in Figure 1 , it can be observed that the same cortical areas are being activated.

tened representation (Engel et al., 1997). The TS of each voxel underwent the following analysis: (1) the linear trend over the 84 temporal samples was removed; (2) the TS was divided by the mean intensity of the voxel; (3) Fourier analysis was applied to obtain the amplitude and phase for each frequency; and (4) the coherence $(c)$ with a sinusoid with a frequency equal to that of the visual stimulation $(1 / 36 \mathrm{~Hz})$ was calculated (Engel et al., 1997). The representations of phase on the flattened cortex were then inspected for "phase reversal," which define the boundaries of visual areas. Examples of these "flat maps" (right hemisphere only) with phase represented in pseudocolor are shown in Figure $2 a$ for two of the participants in the study. The phase reversals/visual area boundaries are indicated by the dotted lines.

Area V3A is a visual region that contains a full hemifield representation of the contralateral visual field (Tootell et al., 1997), which differentiates it from dorsal and ventral V2 and V3, which map only a quadrant of the contralateral field. This hemifield representation, which demarcates V3A in the two subjects' flat maps (Fig. 2a), is shown on their respective structural MRIs in Figure $2 b$. Comparison with the activations generated by the motion stimulus in the bottom panels of Figure 1 in the same subjects shows a near-exact correspondence between the functional and retinotopic identifications of V3A. This provides verification that the area that we will be stimulating in the superior occipito-parietal region is indeed V3A. Table 1 lists the Talairach coordinates for the regions identified as V5 and V3A in the right hemisphere for each of the observers. The locations are consistent with previously published data for the locations of V5 and V3A (Watson et al., 1993; DeYoe et al., 1996; Tootell et al., 1997; Chawla et al., 1998; Rees et al., 2000; Zeki et al., 2003).

Coregistration of FMRI and TMS coil. Localization of the TMS coil with respect to prespecified functional areas of interest (V5/MT + and V3A) was achieved following the methodology described by Sack et al. (2006) using a threedimensional ultrasound digitizer CMS30P (Zebris) in conjunction with the BrainVoyager QX software. Structural and functional MRI scans were coregistered with the subject's head by creating a local spatial coordinate system linking the relative raw spatial position of the ultrasound transmitters with three prespecified anatomical landmarks using the digitizer pen. The prespecified landmarks used were the nasion and the two incisurae intertragicae. The points were then coregistered with the same predefined anatomical points on the head representation (mesh) of the subject. Similarly, a local coordinate system was set up for the TMS coil by linking the prespecified points of the coil with the ultrasound transmitters. Once such coregistration had taken place, movement of the coil with respect to the subject's head is registered and visualized in real time using the Brainvoyager QX software. The coil could then be navigated over specific areas of activation with accuracy and maintained on a trial-by-trial basis.

Stimuli and psychophysical procedure. Stimuli were generated on a high-resolution color graphics monitor with a refresh rate of $120 \mathrm{~Hz}$ (GDM500; Sony), controlled via a VSG graphics card (VSG 2/3, V.5; Cambridge Research Systems). Stimuli had a mean luminance of $12.5 \mathrm{~cd} /$ $\mathrm{m}^{2}$; this was equal to the background luminance of the screen. Subjects viewed a fixation cross with their right eye at a distance of $114 \mathrm{~cm}$. rTMS was always applied to the right hemisphere, and during conditions involving stimulation of cortical areas V3A and V5/MT+, the visual stimuli were horizontally displaced by $15^{\circ}$ to the left of the fixation point (Fig. 3). This protocol was adopted to ensure involvement of right V5/MT+ in task performance while at the same time minimizing any potential contribution from left V5/ $\mathrm{MT}+$ in the opposite cerebral hemisphere. In control experiments, we varied the position of the stimuli and found larger effects for stimuli offset from central fixation. For rTMS delivery to V1, visual stimuli were located at the fixation point. This is because the representation of the central visual field lies closest to the occipital pole and is amenable to disruption by TMS. More eccentric visual field locations are represented deeper within the calcarine fissure. In the absence of reliable data as to the degree of attenuation of the effects of rTMS with increasing cortical depth, we opted for central placement of the stimulus and stimulation of the central field representation in an attempt to maximize the potential effects of TMS. Control experiments showed that varying stimulus position had little influence on the behavioral effects induced by rTMS delivery to V1.

The moving stimuli were vertically orientated, $1 \mathrm{c} /{ }^{\circ}$ sinusoidal luminance gratings, with a contrast of $50 \%$ presented in a square window of side $2.5^{\circ}$. The reference had a speed of $8^{\circ} / \mathrm{s}$ while the test moved at one of seven predetermined speeds, designated to span a range above and below the reference speed. A 2AFC paradigm was used and the observers' task was to indicate which of the two temporally separated gratings appeared to move faster, the reference or the test, by pushing an appropriate switch on a response box. The reference and test grating were each presented for $200 \mathrm{~ms}$, separated by an interstimulus interval (ISI) of $1250 \mathrm{~ms}$. The train of repetitive TMS pulses could be applied at any point within the timeline shown in Figure 3. Three experimental blocks, incorporating 10 trials per test speed, were undertaken, and the data were fitted by a standard psychometric function (Eq. 1) to determine the effect of TMS on the point of subjective equality (PSE) and discrimination threshold as follows: 


$$
y=100-\frac{100}{1+e^{-\frac{(x-\mu)}{\theta}}},
$$

where $y$ is the percentage of times the test was judged as moving faster than the reference, $x$ is the speed of the test stimulus, $\mu$ is the speed corresponding to the $50 \%$ level on the psychometric function (i.e., PSE), and $\theta$ is an estimate of the speed matching threshold. This final value $(\theta)$ was divided by the reference speed to give a Weber fraction for speed discrimination $(\Delta v / v)$.

TMS protocol. A train of five biphasic TMS pulses was applied to the subject's scalp using a figure-of-eight coil (70 $\mathrm{mm}$ diameter) connected to a Magstim Rapid stimulator. The coil was secured in a tripod and placed over the scalp tangentially with the handle orientated upward. The position of the coil was monitored in real time with respect to the subject's head and could be accurately positioned over specific cortical areas of interest (e.g., V5/MT+, V3A, V1). The rTMS pulses were applied at a frequency of $25 \mathrm{~Hz}$, over $0.2 \mathrm{~s}$, typically at a level of $75 \%$ of the maximum output, although this was varied in control experiments (Fig. 3 ). Observers undertook six blocks of trials during any one experimental session. Experimental blocks were organized so that the train of TMS pulses could be initiated at one of 11 temporal positions within the trial. Within any one block, the onset of rTMS was fixed but could be varied in temporal location between blocks. These temporal positions were $200 \mathrm{~ms}$ prereference onset, at reference onset and offset, at 42, 83, 167, 333, 667, and $1000 \mathrm{~ms}$ postreference offset, and at test onset and offset.

Participants. Five subjects (mean age, 31.5 years; range, 23-40 years) participated in this study, four of whom were authors plus one subject who was naive to the aims of the study (N.C.). All had normal visual fields and were corrected to $6 / 5$ with a negative history of psychiatric and neurological disorders. Participants were informed with regard to possible health risks when undertaking the fMRI and TMS procedure, completed a questionnaire detailing contraindications, and gave their informed consent. Experiments were given approval by the local ethics committees (University of Bradford and University of York) and were conducted in accordance with the Declaration of Helsinki and accepted TMS safety protocols (Wassermann, 1998).

\section{Results}

\section{rTMS at test onset}

In the first series of experiments, rTMS stimulation was temporally coincident with the onset of the test stimulus. The TMS coil was placed over the visual area $\mathrm{V} 1, \mathrm{~V} 3 \mathrm{~A}$, or $\mathrm{V} 5 / \mathrm{MT}+$, under the real-time guidance of the neuronavigation system, to examine how stimulation of these areas might differentially affect observer performance on the speed discrimination task. Figure $4 a$ shows the psychometric functions obtained from two observers when rTMS was applied to these areas. Also shown in the plots are the functions obtained under conditions when no TMS was applied.

The delivery of rTMS to areas V3A and V5/MT+ generates two main effects. First, there is a shift in the psychometric functions to the right (i.e., an increase in the PSE), which indicates that under the influence of TMS stimulation the test stimulus is perceived as moving more slowly that the reference. The groupaveraged results for the effects of these conditions on the PSEs and speed discrimination thresholds are shown in Figure 4, $b$ and c. Across the group, a repeated-measures ANOVA shows this increase in PSE to be significantly different from the baseline condition (i.e., no TMS) for both V3A $\left(F_{(1,4)}=161.369 ; p<\right.$ $0.001)$ and $\mathrm{V} 5 / \mathrm{MT}+\left(F_{(1,4)}=39.65 ; p<0.005\right)$. In addition to inducing a perceived slowing of the test stimulus, the application of TMS brings about increases in speed discrimination thresholds $(\Delta v / v)$ revealed by changes in the slopes of the psychometric

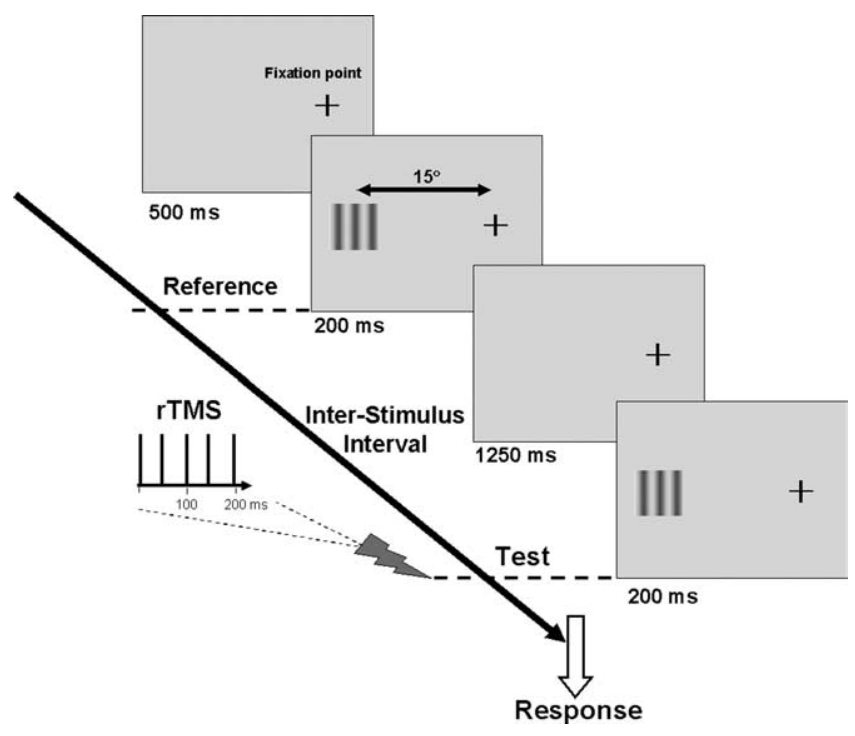

Figure 3. Timeline of experimental paradigm. The temporal sequence of stimulus presentation used in the delayed speed discrimination paradigm. The moving reference and test stimuli (for full details, see Materials and Methods) were presented $15^{\circ}$ to the left of the fixation point (note that for rTMS stimulation of V1, both the reference and test stimulus were placed at the fixation point) and were randomly jittered in terms of motion direction (leftward or rightward), spatial phase on a trial-by-trial basis. These stimuli were separated by an ISI of $1250 \mathrm{~ms}$. In this example, the delivery of the rTMS pulses is coincident with the onset of the test stimulus but could be placed at any point along the timeline.

functions. When rTMS is applied to V5/MT+ and V3A the induced increases in speed discrimination threshold just reach statistical significance when compared to the baseline (no-TMS) condition $\left(\mathrm{V} 5 / \mathrm{MT}+: F_{(1,4)}=7.76, p=0.05\right.$; V3A: $F_{(1,4)}=7.67$, $p=0.05)$. In comparison, rTMS stimulation of area V1 during the performance of the speed discrimination task generates little observable effect on the psychometric function and is not significantly different in terms of PSE or discrimination threshold from that obtained in the baseline condition.

To make any claims about the functional roles of areas V5/ $\mathrm{MT}+$ and V3A in relation to the perception of speed, it is necessary to demonstrate that the effects reported above are specific to the task as well as cortical location over which the rTMS pulse train is delivered. In control experiments, stimulation of $\mathrm{V} 3 \mathrm{~A}$ and $\mathrm{V} 5 / \mathrm{MT}+$ was repeated for three of the observers during performance on a spatial frequency discrimination task. The data are shown in Figure 5, the psychometric curves on the left (Fig. 5a) and the PSEs (Fig. 5b) and spatial frequency discrimination thresholds (Fig. $5 c$ ) on the right. A repeated-measures ANOVA revealed no significant differences between the conditions on ei- 
a)
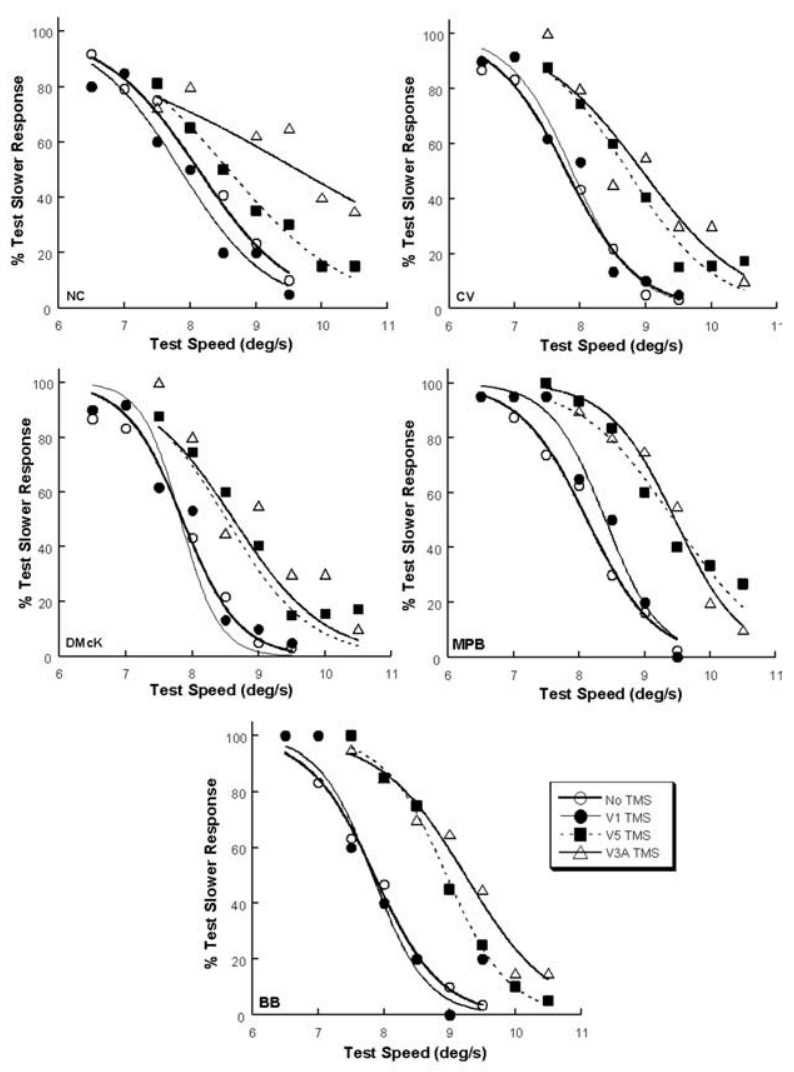

b)

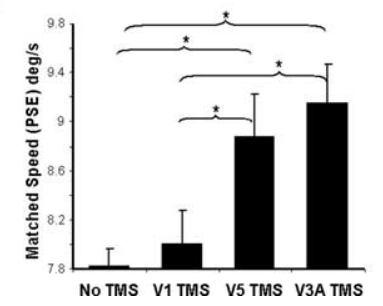

c)

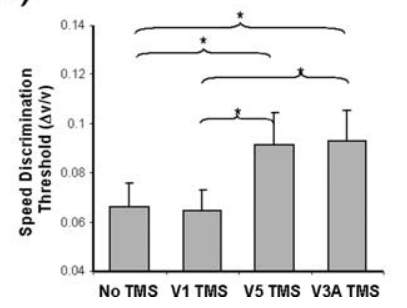

Figure 4. Effect of rTMS on performance in speed discrimination task. $\boldsymbol{a}$, Psychometric functions showing the performance of all five participants on the speed discrimination paradigm. The different curves indicate how performance is affected by the application of rTMS to areas V5/MT +, V3A, and V1 compared with performance when no TMS was delivered. The actual speed of the reference grating was $8 \%$. TMS had little effect when applied to V1. When applied to V5/MT + and V3A, the psychometric functions are shifted to the right, indicating perceived slowing of the test stimulus induced by TMS. The functions also exhibit decreases in slope, which signify an elevation in speed discrimination threshold. $\boldsymbol{b}, \boldsymbol{c}$, Group-averaged data $(n=5)$ showing the mean PSE values and speed discrimination thresholds $(\Delta v / v)$ for the different experimental conditions. The asterisks indicate comparisons for which there are significant differences $(p<0.05)$ between the no-TMS and applied-TMS conditions. The error bars in this and all subsequent figures refer to $\pm 1 S D$ of the mean.

ther the PSE or discrimination threshold. This demonstrates that there is indeed a degree of selectivity in terms of the type of task affected by the application of TMS to areas V3A and V5/MT+. This control also rules out the possibility that any reduction in performance in the speed matching task may have been attributable to blinks generated by stimulation of facial nerves or any other nonspecific effects of TMS stimulation.

To examine the spatial specificity of the effects of TMS, we performed additional experiments during which rTMS was applied to areas of the scalp overlying regions adjacent to areas $\mathrm{V} 5 / \mathrm{MT}+$ and V3A while observers performed the main speed a)
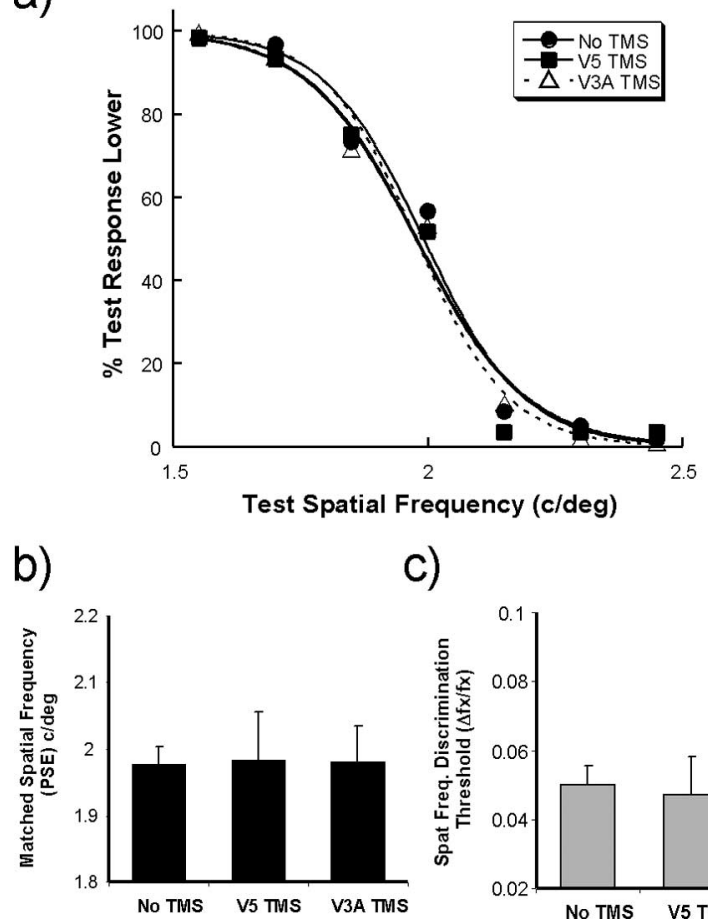

c)

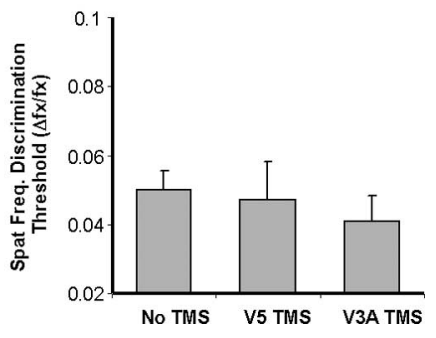

Figure 5. Task specificity control. $\boldsymbol{a}$, Group-averaged psychometric functions showing how performance varied on a spatial frequency discrimination task while rTMS was delivered to areas V3A and V5/MT+. $\boldsymbol{b}, \boldsymbol{c}$, Performance is also shown for the no-TMS condition. The mean PSEs $(\boldsymbol{b})$ and spatial frequency discrimination thresholds $(\Delta f / f)(\boldsymbol{c})$ are also shown for the three experimental conditions, none of which exhibit any significant differences from one another.

discrimination task. Controls for the lateral spread of TMS effects are commonly performed by moving the coil $1-2 \mathrm{~cm}$ away from the primary site of stimulation. In this experiment, we targeted the TMS to prominent features of the individuals' sulcal and gyral anatomy, close $(2 \mathrm{~cm})$ to the functionally identified areas of V5/ $\mathrm{MT}+$ and V3A, but which retinotopic procedures identified as lying outside these areas. Results from these experiments are shown in Figure 6. In the top panel (Fig. 6a), a site posterior and medial to V5/MT+ on the surface of the occipito-temporal cortex was targeted. The effect of TMS delivery to this region of the cortex on the psychometric function is shown in Figure $6 b$. Also shown in Figure $6 b$ are the functions obtained when V5/MT was targeted and when no TMS was delivered. The effect of changing the cortical site of TMS delivery produces a function that induces no apparent effects in matched speed or discrimination threshold from the no-TMS condition. In the bottom panels (Fig. $6 c, d$ ), the effects of TMS delivery to a site close to V3A are shown for a different observer. In this case, the site of TMS application is ventral to $\mathrm{V} 3 \mathrm{~A}$, lying in a region that the retinotopic mapping procedures for this observer indicate is dorsal V3. Once again, shifting the point of application of the rTMS away from V3A affects performance in a similar manner as the shift away from $\mathrm{V} 5 / \mathrm{MT}+$ with performance becoming similar to that achieved in the no-TMS condition, as indicated by the functions shown in Figure $6 d$.

Finally, to firmly establish the link between our TMS application and the induced deficits in speed perception reported above, in another control experiment we examined the effect of changing the strength of the rTMS pulses on behavioral performance. By measuring the effects on speed matching of decreasing TMS strength, we demonstrated a correlation between the magnitude 
of the pulse delivered and its effect on performance. Figure 7 plots the effect of varying TMS strength on the PSE and speed discrimination threshold for V5/MT+ and V3A stimulation for three observers. Across the range tested (30-75\% of maximum output), the decreases in performance, as measured by perceived slowing of the test stimulus and increased speed discrimination thresholds, are well described by linear functions (PSEs: V5/MT, $r^{2}=0.948$; V3A, $r^{2}=0.951$; discrimination thresholds: V5/MT, $r^{2}=0.761$; V3A, $\left.r^{2}=0.952\right)$, thus establishing a correlation between the strength of the applied TMS and induced behavioral deficits.

\section{rTMS at different stimulus onset asynchronies}

The temporal relationship between the delivery of TMS pulses and the appearance of motion stimuli has often been emphasized with studies indicating that the effects of TMS on motion perception are greatest when pulses are applied within certain temporal windows (Hotson et al., 1994; Beckers and Zeki, 1995; Sack et al., 2006). So far, we have only considered the effects of TMS when its delivery is coincident with the onset of the test stimulus. In the light of previous findings, we examined how varying the timing of the onset of rTMS pulses during the stimulus delivery cycle would affect observer performance on the speed discrimination task. Figure 8 shows data (average of five observers) from such an experiment in which rTMS was applied to V5/MT+, V3A, or V1 at different temporal locations spanning the reference stimulus foreperiod, ISI, and test offset. Again, we were interested in the influence of the rTMS delivery on both the PSEs and speed discrimination thresholds, which are plotted in Figure 8, $a$ and $b$, respectively.

When the onset time of the rTMS to area V1 is varied over the reference/test stimulus delivery cycle, there is a minimal effect on perceived speed. At all timings, the mean matched test speed is within \pm 1 SD for that obtained for the baseline condition (dotted line) when no TMS was applied. However, when rTMS was applied to regions of the scalp overlying areas V5/MT+ and V3A, the effect on matching speed was greatest when the pulse train was temporally coincident with test stimulus onset. The speed of the test has to be effectively increased to counteract the perceived slowing induced by the rTMS, in order that a match with the reference stimulus can be obtained. Interestingly, the exact opposite effect can be observed when the onset rTMS pulse train is coincident with the reference onset. In this case, it is the reference stimulus that is perceived as moving more slowly under the influence of the TMS and the speed of the test stimulus is reduced below that of the baseline condition to match the reference. Figure $8 a$ also shows that, as the temporal interval between the onset of the rTMS pulse train and the onset of the test stimulus increases, the effect on its perceived speed diminishes. When the rTMS onset precedes the test onset by $\sim 900 \mathrm{~ms}$, the PSEs ob- tained are not significantly different from those obtained in the no-TMS condition.

Although the effects of rTMS delivery to V5/MT+ and V3A on perceived speed of the matched test stimulus were very repeatable across subjects, the induced changes in speed discrimination thresholds were much more variable. Some participants exhibited large increases in discrimination threshold when V5/MT+ and V3A were stimulated, others exhibited very little at all. This greater intersubject variability is revealed by the much larger error bars shown for the discrimination data in Figure $8 b$. The only point in the stimulus delivery cycle at which the application of rTMS significantly affects discrimination thresholds is at the onset of the test stimulus (see above). Although the group-averaged data do appear to show an induced maximum in threshold when rTMS is applied to area V5/MT $+200 \mathrm{~ms}$ after the onset of the reference stimulus, this increase fails to reach a level that is significantly different $\left(F_{(1,4)}=4.825 ; p=0.093\right)$ from the no-TMS condition.

\section{Discussion}

This study demonstrates that human speed perception can be selectively and reversibly impaired when rTMS is carefully targeted so as to disrupt the normal functioning of cortical areas $\mathrm{V} 5 / \mathrm{MT}+$ and V3A. The deficits induced by rTMS to these areas are both task and location specific. In addition, the magnitude of 
a)

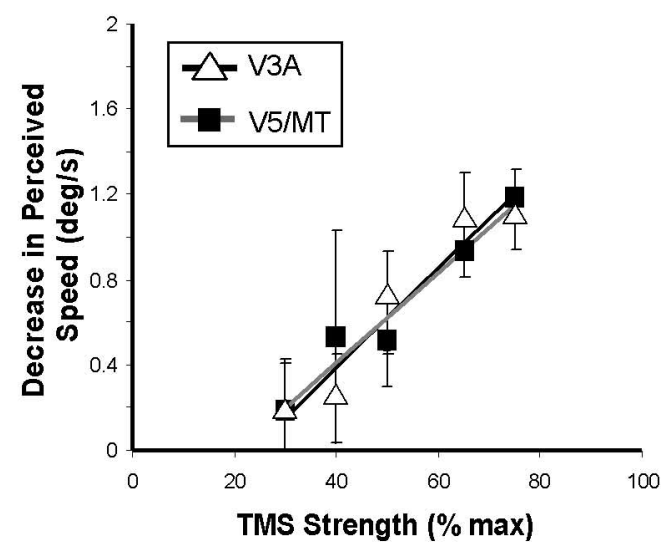

b)

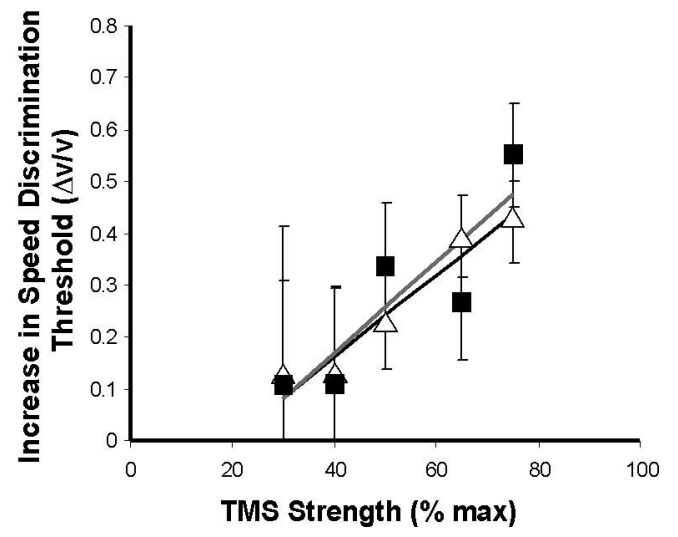

Figure 7. Effect of varying TMS strength. $\boldsymbol{a}$, The magnitude of the decrease in perceived test stimulus speed plotted as a function of rTMS strength. Data are shown for application of rTMS to both V5/MT + and V3A and are the average of three observers. The data have been fitted with linear regression lines with correlation coefficients $\left(r^{2}\right)$ of 0.948 for the V3A data and 0.951 for the V5/MT+ data. $\boldsymbol{b}$, Similar plots to $\boldsymbol{a}$ showing the increase in speed discrimination thresholds $(\Delta v / v)$ with increasing TMS strength; the correlation coefficients $\left(r^{2}\right)$ in this case are 0.952 for V3A and 0.761 for V5/MT+.

the deficits is correlated with the strength of the applied magnetic stimulation.

\section{Cortical areas involved in speed perception}

The fact that disruption to area V3A can generate impairments in speed perception that are of a similar magnitude to those induced when V5/MT + is disrupted, represents a significant finding. Hitherto, V5/MT has been considered as the main cortical locus for the neural mechanisms that underpin the perception of stimulus speed (Newsome and Paré, 1988; Orban et al., 1995; Churchland and Lisberger, 2001; Perrone and Thiele, 2001; Priebe et al., 2003; Priebe and Lisberger, 2004; Liu and Newsome, 2005, 2006; Nover et al., 2005; Krekelberg et al., 2006a,b). The results presented here demonstrate that not only is behaviorally relevant information concerning stimulus speed encoded by activity in human area V5/MT+, but that it is also encoded by activity within area V3A. The fact that we can induce deficits in perception by applying rTMS to area $\mathrm{V} 3 \mathrm{~A}$ indicates that information about speed is available at this level and would appear to cement the prominent status of V3A in the cortical network that exists for the processing of motion in the human brain (Tootell et al., 1997; Smith et al., 1998; Seiffert et al., 2003; Liu and Wandell, 2005).

The application of rTMS to cortical areas V3A and V5/ $\mathrm{MT}+$ induces a perceived slowing of presented moving stim- a)

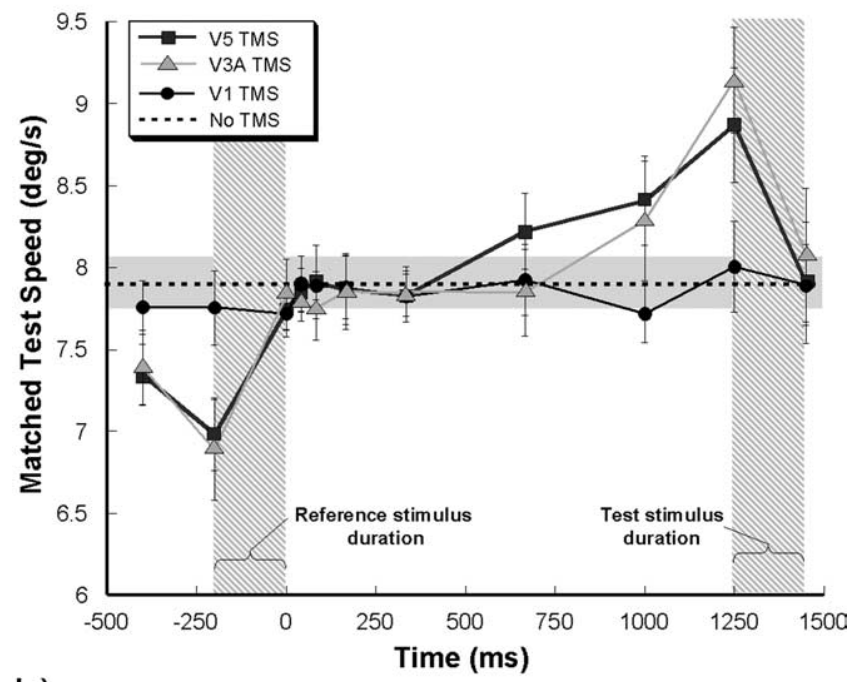

b)

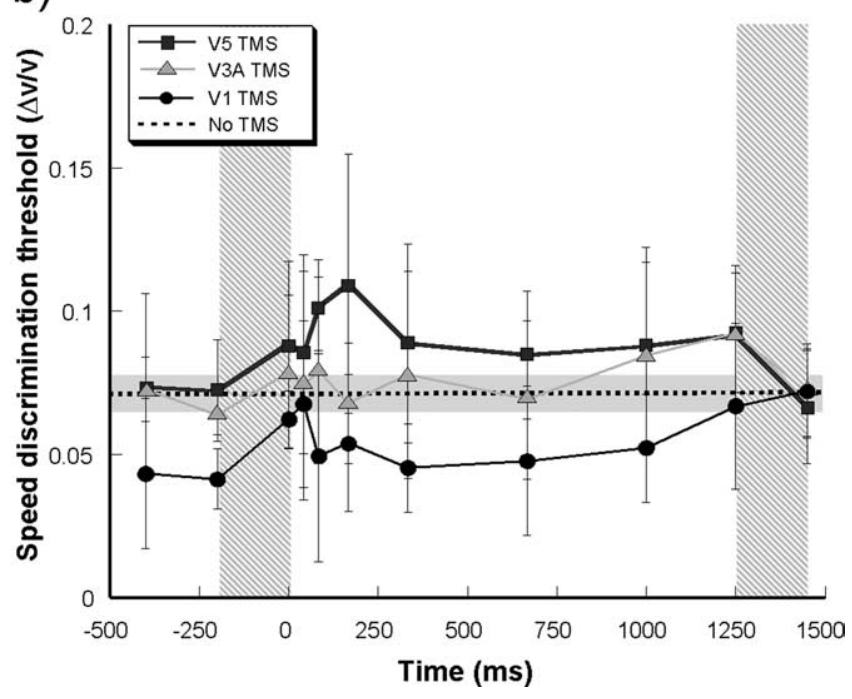

Figure 8. TMS pulse timing. $\boldsymbol{a}$, The variation in the speed of the test stimulus that was required to match an $8 \%$ r reference when the onset of the rTMS was delivered at different times during the stimulus delivery cycle. The reference and test stimulus onset durations are indicated by the vertical shaded blocks. The variation of matched speed (i.e., PSE) is shown for V5/MT+ (black squares/line), V3A (gray triangles/line), and V1 stimulation (black circles/line). The mean matched speed when no TMS was delivered is shown by the horizontal dotted line banded by a gray area that represents $\pm 1 S D$ of the mean. The results represent the average of five subjects. $\boldsymbol{b}$, The variation in speed discrimination thresholds $(\Delta v / v)$ for the same experiment as shown in $\boldsymbol{a}$. The same conventions as above apply.

uli and elevates speed discrimination thresholds $(\Delta v / v)$. These deficits in speed perception are consistent with the findings of Matthews et al. (2001) who reported similar effects for singlepulse TMS application in the vicinity of human V5/MT+. However, although the nature of the effects might be consistent across the two studies, the precise pattern of deficits elicited as a function of TMS application site differ markedly. For example, Matthews et al. report a greater deficit in speed discrimination when TMS is applied close to area V1, at a medial site lying $2 \mathrm{~cm}$ above the inion, compared with when it is applied at a more lateral site $(5 \mathrm{~cm}$ lateral to and $4 \mathrm{~cm}$ above the inion), which was chosen because of its reported proximity to human area V5/MT + . Our results show the opposite effect, with greater deficits in speed discrimination thresholds pro- 
duced when rTMS is applied over V5/MT + , and little or no change in performance, compared with the no-TMS condition, when TMS is applied over V1. In addition, Matthews et al. (2001) show that the "perceived slowing" effect that the delivery of TMS has on a moving stimulus is of a similar magnitude for both medial (i.e., V1) and lateral (i.e., V5/ $\mathrm{MT}+$ ) stimulation. In this study, we find this effect to be greatest when rTMS is applied to areas V3A and V5/MT+, whereas for V1 stimulation the perceived speed was found not to be significantly different from the no-TMS condition. It is not clear to us what the reasons are for these discrepant findings. One possibility might lie in the inaccurate localization of areas $\mathrm{V} 1$ and V5/MT+ on the basis of external anatomical landmarks of the skull. Visual areas are known to vary in location and extent across individuals (Watson et al., 1993; Tootell et al., 1997; Smith et al., 1998), and the results from Figure 6 give an indication as to how localized the effects of TMS can be. Therefore, in the absence of functional or retinotopic neuroimaging data, it is difficult to say with any certainty precisely which visual areas were stimulated in previous studies. Nonetheless, the absence of deficits in speed perception after the delivery of rTMS to V1 is surprising, not least because $\mathrm{V} 1$ is a major source of direct input into area V5/MT (Shipp and Zeki, 1989). However, we must be careful not to over interpret this finding as indicating a minimal role for $\mathrm{V} 1$ in motion processing. On the contrary, previous TMS studies have highlighted the importance of $\mathrm{V} 1$, and its reciprocal connectivity with $\mathrm{V} 5 / \mathrm{MT}+$, in the visual awareness of motion (Pascual-Leone and Walsh, 2001). Instead, the results may indicate that the contribution of $\mathrm{V} 1$ to this particular speed task is not crucial, despite recent reports of speed tuned neurons in this area (Priebe et al., 2006). Alternatively, the absence of speed deficits after TMS of V1 may indicate that motion signals used in the computation of speed can bypass V1, reaching V5/MT either via direct connections with the LGN (Sincich et al., 2004) or via the pulvinar, which is known to contain neurons responsive to motion (Merabet et al., 1998; Villeneuve et al., 2005).

\section{The effects of rTMS on speed perception and discrimination} Our results demonstrate that the application of TMS to areas $\mathrm{V} 5 / \mathrm{MT}+$ and V3A always leads to a decrease in perceived speed, never an increase. In this respect, our results are opposite to those that have been reported for microstimulation studies. For example, Komatsu and Wurtz (1989) have shown that electrical stimulation of monkey V5/MT neurons leads to an increase in perceived speed (as evidenced by increases in the speed of pursuit eye movements), with larger injected currents generating increases of larger magnitude. The fact that our observers always report a decrease in speed would therefore seem to imply that the delivery of rTMS to areas V5/MT+ and $\mathrm{V} 3 \mathrm{~A}$, rather than inducing an increase in neural activity, as in the case of microstimulation, leads to decreased neural activity in these areas. Although being cautious about the comparison of TMS and microstimulation techniques, which affect very different numbers of cortical neurons, our findings are nonetheless consistent with recent ideas put forward by Krekelberg et al. (2006b) regarding the nature of the neural code for speed. They propose that speed is proportional to the total activity of a subpopulation of V5/MT neurons with greater activity signaling faster speeds. However, the induced slowing effect of TMS runs counter to what might be predicted from models of speed perception that are based on vector averaging across neurons that have preferred speeds to which they are optimally responsive, so-called "labeled lines" (Churchland and Lisberger, 2001; Priebe and Lisberger, 2004). If perceived speed was indeed based on some form of weighted average from these labeled lines, then the disruption of neural activity by TMS would be expected to reduce the activity across all preferred responses indiscriminately; the net result being that there would be no effect on behavior (Krekelberg et al., 2006b).

The notion that rTMS delivery can bring about a reduction in the response from V5/MT that signals stimulus speed is consistent with ideas that suggest that TMS works via the suppression of neural signal relating to the target stimulus (Harris et al., 2008). Physiological recordings from the cat visual cortex give direct support for this view (Allen et al., 2007) with the suppression of activity leading to a decrease in the signal-tonoise $(\mathrm{S}: \mathrm{N})$ ratio from the cortical area to which TMS is delivered (Walsh and Cowey, 2000; Cowey, 2005). Another view is that TMS may also work by decreasing S:N ratio via an increase in the background noise (Walsh and Cowey, 2000; Silvanto et al., 2007a,b). The elevations of speed discrimination thresholds indicate that, as well as suppressing activity relating to the stimulus, noise is also being added via the application of rTMS. One potential mechanism for this might be via the addition of increased background spontaneous activity, which also occurs when TMS is applied to the visual cortex (Allen et al., 2007). Human speed judgments are very precise and observers can typically obtain velocity Weber fractions $(\Delta v / v)$ of the order of 0.05 for a wide range of stimulus speeds (McKee et al., 1986). This performance reflects the signal-to-noise characteristics of the underlying physiological mechanisms and any factor that increases the noise should therefore degrade discriminative ability. This is exactly what the targeted delivery of rTMS to V5/MT+ and V3A does. Performance in delayed discrimination tasks of this kind used in this study are thought to be based on the neural representation, or buildup, of some implicit internal standard or template (Morgan et al., 2000; Kahana and Sekuler, 2002). One possibility is that the application of TMS may degrade this representation of the reference stimulus, which is retained by short-term visual memory mechanisms. Thus, the effects of TMS application to $\mathrm{V} 5 / \mathrm{MT}+$ and V3A during our delayed speed discrimination task, suggests that these cortical regions might be important for the retention of sensory information about motion in the human brain. Certainly, there is evidence to suggest that V5/MT neurons are involved in this kind of role (Bisley and Pasternak, 2000). In addition, perceptual priming in motion direction discrimination tasks can also be abolished by the application of TMS to human V5/MT+ (Campana et al., 2002). As yet however, we are not aware of any evidence that shows that V3A might also be involved in the retention of information about the speed of moving stimuli, something we want to explore in future experiments.

\section{References}

Allen EA, Pasley BN, Duong T, Freeman RD (2007) Transcranial magnetic stimulation elicits coupled neural and hemodynamic consequences. Science 317:1918-1921.

Anand S, Olson JD, Hotson JR (1998) Tracing the timing of human analysis of motion and chromatic signals from occipital to tempero-parietooccipital cortex: a transcranial magnetic stimulation study. Vision Res 38:2619-2627.

Beckers G, Hömberg V (1992) Cerebral visual motion blindness: transitory 
akinetopsia induced by transcranial magnetic stimulation of human area V5. Proc Biol Sci 249:173-178.

Beckers G, Zeki S (1995) The consequences of inactivating areas V1 and V5 on visual motion perception. Brain 118:49-60.

Bisley JW, Paternak T (2000) The multiple roles of cortical areas MT/MST in remembering the direction of visual motion. Cereb Cortex 10:1053-1065.

Boynton GM, Engel SA, Glover GH, Heeger DJ (1996) Linear systems analysis of functional magnetic resonance imaging in human V1. J Neurosci 16:4207-4221.

Campana G, Cowey A, Walsh V (2002) Priming of motion direction and area V5/MT: a test of perceptual memory. Cereb Cortex 12:663-669.

Chawla D, Phillips J, Buechel C, Edwards R, Friston KJ (1998) Speeddependent motion-sensitive responses in V5: an fMRI study. Neuroimage 7:86-96.

Churchland MM, Lisberger SG (2001) Shifts in the population response in the middle temporal visual area parallel perceptual and motor illusions produced by apparent motion. J Neurosci 21:9387-9402.

Cowey A (2005) The Ferrier Lecture: What can transcranial magnetic stimulation tell us about how the brain works? Philos Trans R Soc Lond B Biol Sci 360:1185-1205.

Cowey A, Campana G, Walsh V, Vaina LM (2006) The role of human extrastriate visual areas V5/MT and V2/V3 in the perception of the direction of global motion: a transcranial magnetic stimulation study. Exp Brain Res 171:558-562.

DeYoe EA, Carman GJ, Bandettini P, Glickman S, Wieser J, Cox R, Miller D, Neitz J (1996) Mapping striate and extra-striate visual areas in human cerebral cortex. Proc Natl Acad Sci U S A 93:2382-2386.

Dupont P, Orban GA, De Bruyn B, Verbruggen A, Mortelmans L (1994) Many areas in the human brain respond to visual motion. J Neurophysiol $72: 1420-1424$.

Engel SA, Glover GH, Wandell BA (1997) Retinotopic organization in human visual cortex and the spatial precision of functional MRI. Cereb Cortex 7:181-192.

Harris JA, Clifford CW, Miniussi C (2008) The functional effect of transcranial magnetic stimulation: signal suppression or neural noise generation? J Cogn Neurosci 20:734-740.

Hotson J, Braun D, Herzberg W, Boman D (1994) Transcranial magnetic stimulation of extra-striate cortex degrades human motion direction discrimination. Vision Res 34:2115-2123.

Huk AC, Heeger DJ (2000) Task-related modulation of visual cortex. J Neurophysiol 83:3525-3536.

Kahana MJ, Sekuler R (2002) Recognizing spatial patterns: a noisy exemplar approach. Vision Res 42:2177-2192.

Komatsu H, Wurtz RH (1989) Modulation of pursuit eye movements by stimulation of cortical areas MT and MST. J Neurophysiol 62:31-47.

Krekelberg B, van Wezel RJ, Albright TD (2006a) Adaptation in macaque MT reduces perceived speed and improves speed discrimination. J Neurophysiol 95:255-270.

Krekelberg B, van Wezel RJ, Albright TD (2006b) Interactions between speed and contrast tuning in the middle temporal area: implications for the neural code for speed. J Neurosci 26:8988-8998.

Lagae L, Raiguel S, Orban GA (1993) Speed and direction sensitivity of macaque middle temporal neurons. J Neurophysiol 69:19-39.

Laycock R, Crewther DP, Fitzgerald PB, Crewther SG (2007) Evidence for fast signals and later processing in human V1/V2 and V5/MT+: a TMS study of motion perception. J Neurophysiol 98:1253-1262.

Liu J, Newsome WT (2005) Correlation between speed perception and neural activity in the middle temporal visual area. J Neurosci 25:711-722.

Liu J, Newsome WT (2006) Local field potential in cortical area MT: stimulus tuning and behavioral correlations. J Neurosci 26:7779-7790.

Liu J, Wandell BA (2005) Specializations for chromatic and temporal signals in human visual cortex. J Neurosci 25:3459-3468.

Matthews N, Luber B, Qian N, Lisanby SH (2001) Transcranial magnetic stimulation differentially affects speed and direction judgments. Exp Brain Res 140:397-406.

Maunsell JH, Van Essen DC (1983) Functional properties of neurons in the middle temporal visual area of the macaque monkey. J Neurophysiol 49:1127-1147.

McKee SP, Silverman GH, Nakayama K (1986) Precise velocity discrimination despite random variations in temporal frequency and contrast. Vision Res 26:609-619.
McKeefry DJ, Watson JD, Frackowiak RS, Fong K, Zeki S (1997) The activity in human areas V1, V2, V3 and V5 during the perception of coherent and incoherent motion. Neuroimage 5:1-12.

Merabet L, Desautels A, Minville K, Casanova C (1998) Motion integration in a thalamic visual nucleus. Nature 396:265-268.

Morgan MJ, Watamaniuk SN, McKee SP (2000) The use of an implicit standard for measuring discrimination thresholds. Vision Res 40:2341-2349.

Newsome WT, Paré EB (1988) A selective impairment of motion perception following lesions of the middle temporal visual area (MT). J Neurosci 8:2201-2211.

Nover H, Anderson CH, DeAngelis GC (2005) A logarithmic, scaleinvariant representation of speed in macaque middle temporal area accounts for speed discrimination performance. J Neurosci 25:10049-10060.

Orban GA, Saunders RC, Vandenbussche E (1995) Lesions of the superior temporal cortical motion areas impair speed discrimination in the macaque monkey. Eur J Neurosci 7:2261-2276.

Pascual-Leone A, Walsh V (2001) Fast backprojections from the motion to the primary visual area are necessary for visual awareness. Science 292:510-512.

Pascual-Leone A, Walsh V, Rothwell J (2000) Transcranial magnetic stimulation in cognitive neuroscience-virtual lesion, chronometry and functional connectivity. Curr Opin Neurobiol 10:232-237.

Perrone JA, Thiele A (2001) Speed skills: measuring the visual speed analyzing properties of primate MT neurons. Nat Neurosci 4:526-532.

Priebe NJ, Lisberger SG (2004) Estimating target speed from the population response in visual area MT. J Neurosci 24:1907-1916.

Priebe NJ, Cassanello CR, Lisberger SG (2003) The neural representation of speed in macaque area MT/V5. J Neurosci 23:5650-5661.

Priebe NJ, Lisberger SG, Movshon JA (2006) Tuning for spatiotemporal frequency and speed in directionally selective neurons of macamque striate cortex. J Neurosci 26:2941-2950.

Rees G, Friston K, Koch C (2000) A direct quantitative relationship between the functional properties of human and macaque V5. Nat Neurosci 3:716-723.

Rodman HR, Albright TD (1987) Coding of visual stimulus velocity in area MT of the macaque. Vision Res 27:2035-2048.

Sack AT, Kohler A, Linden DE, Goebel R, Muckli L (2006) The temporal characteristics of motion processing in hMT/V5+: combining fMRI and neuronavigated TMS. Neuroimage 29:1326-1335.

Seiffert AE, Somers DC, Dale AM, Tootell RBH (2003) Functional MRI studies of human visual motion perception: texture, luminance, attention and after-effects. Cereb Cortex 13:340-349.

Sereno MI, Dale AM, Reppas JB, Kwong KK, Belliveau JW, Brady TJ, Rosen BR, Tootell RB (1995) Borders of multiple human visual areas in humans revealed by functional MRI. Science 268:889-893.

Shipp S, Zeki S (1989) The organization of connections between areas V5 and V1 in macaque monkey visual cortex. Eur J Neurosci 1:309-332.

Silvanto J, Muggleton NG, Cowey A, Walsh V (2007a) Neural adaptation reveals state-dependent effects of transcranial magnetic stimulation. Eur J Neurosci 25:1874-1881.

Silvanto J, Muggleton NG, Cowey A, Walsh V (2007b) Neural activation state determines behavioural susceptibility to modified theta burst transcranial magnetic stimulation. Eur J Neurosci 26:523-528.

Sincich LC, Park KF, Wohlgemuth MJ, Horton JC (2004) Bypassing V1: a direct geniculate input to area MT. Nat Neurosci 7:1123-1128.

Smith AT, Greenlee MW, Singh KD, Kraemer FM, Hennig J (1998) The processing of first- and second-order motion in human visual cortex assessed by functional magnetic resonance imaging (fMRI). J Neurosci 18:3816-3830.

Sunaert S, Van Hecke P, Marchal G, Orban GA (2000) Attention to speed of motion, speed discrimination, and task difficulty: an fMRI study. Neuroimage 11:612-623.

Teo PC, Sapiro G, Wandell BA (1997) Creating connected representations of cortical gray matter for functional MRI visualization. IEEE Trans Med Imaging 16:852-863.

Tootell RB, Reppas JB, Kwong KK, Malach R, Born RT, Brady TJ, Rosen BR, Belliveau JW (1995a) Functional analysis of human MT and related visual cortical areas using magnetic resonance imaging. J Neurosci 15:3215-3230.

Tootell RB, Reppas JB, Dale AM, Look RB, Sereno MI, Malach R, Brady TJ, Rosen BR (1995b) Visual motion after-effect in human cortical area 
MT/V5 revealed by functional magnetic resonance imaging. Nature 375:139-141.

Tootell RB, Mendola JD, Hadjikhani NK, Ledden PJ, Liu AK, Reppas JB, Sereno MI, Dale AM (1997) Functional analysis of V3A and related areas in human visual cortex. J Neurosci 17:7060-7078.

Villeneuve MY, Kupers R, Gjedde A, Ptito M, Casanova C (2005) Patternmotion selectivity in the human pulvinar. Neuroimage 28:474-480.

Walsh V, Cowey A (2000) Transcranial magnetic stimulation and cognitive neuroscience. Nat Rev Neurosci 1:73-79.

Walsh V, Ellison A, Battelli L, Cowey A (1998) Task-specific impairments and enhancements induced by magnetic stimulation of human visual area V5. Proc Biol Sci 265:537-543.
Wandell BA, Chial S, Backus BT (2000) Visualization and measurement of the cortical surface. J Cogn Neurosci 12:739-752.

Wassermann EM (1998) Risk and safety of repetitive transcranial magnetic stimulation: report and suggested guidelines from the International Workshop on the Safety of Transcranial Magnetic Stimulation. Electroencephalogr Clin Neurophysiol 108:1-16.

Watson JD, Myers R, Frackowiak RS, Hajnal JV, Woods RP, Mazziotta JC, Shipp S, Zeki S (1993) Area V5 of the human brain: evidence from a combined study using positron emission tomography and magnetic resonance imaging. Cereb Cortex 3:79-94.

Zeki S, Perry RJ, Bartels A (2003) The processing of kinetic contours in the brain. Cereb Cortex 13:189-202. 